\title{
The Effects of a Medial Heel Wedge on the Weight- Bearing Response of Hindfoot Valgus and the Total Weight-Bearing Responses of the Navicular and Talus Bones
}

\author{
Masafumi Maruyama ${ }^{1,2^{*}}$, Arito Yozu ${ }^{2,3}$, Yoshikazu Okamoto ${ }^{4}$, Hitoshi Shiraki ${ }^{5}$ \\ ' Graduate School of Comprehensive Human Science, University of Tsukuba, Ibaraki, Japan \\ ${ }^{2}$ Center for Medical Sciences, Ibaraki Prefectural University of Health Sciences, Ibaraki, Japan \\ ${ }^{3}$ Department of Precision Engineering, The University of Tokyo, Tokyo, Japan \\ ${ }^{4}$ Department of Diagnostic and Interventional Radiology, Faculty of Medicine, University of Tsukuba, Ibaraki, Japan \\ ${ }^{5}$ Faculty of Health and Sports Science, University of Tsukuba, Ibaraki, Japan
}

Received: April 21, 2021

Accepted: July 12, 2021

Published online: July 31, 2021

Keywords:

Heel Wedge

Positional MRI

Weight-Bearing

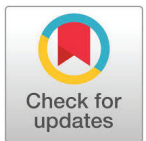

\section{ABSTRACT}

OBJECTIVES Medial heel wedges are commonly prescribed to manage the weight-bearing response of hindfoot valgus and the total weight-bearing responses of the navicular and talus bones. Previous studies have reported that a medial heel wedge is effective in the management of musculoskeletal injuries. However, it remains unclear the effect of a medial heel wedge on the weight-bearing responses of footarch bones in vivo. To clarify the effects of a medial heel wedge on the weight-bearing response of hindfoot valgus and the total weight-bearing responses of the navicular and talus bones is necessary to understand how best to treat musculoskeletal injuries clinically. The purpose of our study was to clarify the effects of a medial heel wedge on the weight-bearing response of hindfoot valgus and the total weight-bearing responses of the navicular and talus bones.

METHODS Twenty-five healthy males were analyzed. We obtained MRI scanning of the right foot under non-loading (NL) and full weight-bearing (FW) conditions. Participants wore two insole types, a flat insole and a medial heel wedge. To evaluate the weight-bearing response in hindfoot valgus, the hindfoot alignment view (HAV) was measured. We also measured navicular and talus bone positions and calculated the total positional changes of the navicular and talus bones ( $\triangle T P C N, \triangle T P C T$ ) from the vertical and medial displacements using the Pythagorean theorem.

RESULTS Significant interactions were observed with the HAV. Under both NL and FW conditions, the HAV was smaller on the medial heel wedge than on the flat insole. In addition, the $\triangle T P C N$ was significantly smaller on the medial heel wedge than on the flat insole. However, no significant differences were observed for $\triangle T P C T$.

CONCLUSIONS Our results suggest that use of a medial heel wedge decreases hindfoot valgus values under both NL and FW conditions and stabilizes the total weight-bearing response of the navicular bone.

( The Asian Society of Kinesiology and the Korean Academy of Kinesiology

*Correspondence: Masafumi Maruyama, Graduate School of Comprehensive Human Science, University of Tsukuba, 1-1-1 Tennodai, Tsukuba, Ibaraki, 305-8574, Japan; Tel: +81-029-853-2575; E-mail: maruyama.masafumi.ws@alumni.tsukuba.ac.jp 


\section{Introduction}

The human foot structure functionally deforms to absorb the impact of the foot [1]. When an axial load is applied, the weight-bearing response of hindfoot valgus and the total weight-bearing responses of the navicular and talus bones are functionally linked together to absorb the load [2-4], which are important processes for absorbing loads [4-6].

On the other hand, an excessive weight-bearing response of hindfoot valgus may apply mechanical stimulation to posterior tendons and cause further microtrauma [7]. Previous studies have reported that an abnormal total weight-bearing response of the navicular and talus bones, such as increased mobility or changes in the ratios of vertical to medial displacement, are likely associated with injuries [8]. Therefore, using foot orthoses to control an excessive weight-bearing response of hindfoot valgus and abnormal total weight-bearing responses of the navicular and talus bones are essential considerations in the clinical setting.

Medial heel wedges are commonly prescribed to manage the weight-bearing responses of foot-arch bones and musculoskeletal injuries [9]. Previous studies have reported that a medial heel wedge is effective in the management of musculoskeletal injuries [10]. Furthermore, previous studies have shown that the use of a medial heel wedge can cause biomechanical changes in the ankle, knee, and hip joints and support the promotion of movement [11-13]. From the abovementioned, it is considered that biomechanical changes in the entire lower limb caused by the use of medial heel wedge contribute to injury prevention [14]. Conversely, the effects of a medial heel wedge on the weight-bearing of foot arch bones are unknown because performing direct measurements of the weight-bearing responses of foot-arch bones in vivo has been challenging. Foot orthosis is prescribed to improve foot function based on the assessment of foot function $[13,15,16]$. Furthermore, previous studies have reported that foot function influences the entire lower limb function $[17,18]$. Therefore, the detailed effects of a medial heel wedge on the weightbearing of foot arch bones will be fundamental knowledge for the management of musculoskeletal injuries of the lower limb.

Recently, positional magnetic resonance imaging (pMRI) has been developed. In a previous study, we investigated the weight-bearing response of hindfoot valgus and the total weight-bearing responses of the navicular and talus bones using pMRI [2]. This method can also be used to determine a medial heel wedge's effectiveness at influencing the weightbearing responses of the foot-arch bones. The present study aimed to clarify the effects of a medial heel wedge on the weight-bearing response of hindfoot valgus and the total weight-bearing responses of the navicular and talus bones.

\section{Methods}

This study was approved by the University of Tsukuba Physical Education Research Institutional Review Board [Task No. PE019-54].

\section{Participants}

Twenty-five male volunteers participated in this study. Participants were recruited from University of Tsukuba students through e-mail and information sharing. All participants were free of lower-extremity pain and subjective symptoms interfering with walking or sports-related activities and had no history of serious injuries or operative treatments. All participants gave written informed consent to the purpose and procedures of this study before participating. The average \pm standard deviation (SD) of the participants' ages, weights, and heights were $23.24 \pm 2.10$ years, $69.04 \pm 5.96 \mathrm{~kg}$, and $173.54 \pm 5.57 \mathrm{~cm}$, respectively.

\section{Protocol}

Our study was comprised of two sessions, including a flat insole session and a medial heel wedge session, for each participant, with at least one day between sessions. Participants wore either the flat insole or the medial heel wedge during the 1st session and then wore the other type of insole during the 2 nd session. This order was randomly assigned. In both sessions, MRI was conducted immediately after the participants wore the insole. During each session, MRI scanning was performed under two conditions: nonloading (NL) and full weight-bearing (FW). 


\section{Insole Types}

The two insoles were a combination of a flat cork insole and silicon heel wedge. A heel wedge was not used for flat insoles, whereas an elevation of approximately $4^{\circ}$ was used for the medial heel wedge. The length, width, and thickness of the medial heel wedge were $5.5 \mathrm{~cm}, 8.0 \mathrm{~cm}$, and $1.0 \mathrm{~cm}$, respectively. A heel wedge was attached to the inner heel section of the flat cork insoles.

\section{Magnetic Resonance Imaging Procedure}

Imaging was conducted using a positional MRI system (0.25 T G-scan, Esaote, Genoa, Italy). We scanned the right foot in two different conditions: NL and FW [19,20]. The applied pMRI protocol included the following sequences: scout-slice thickness, $10 \mathrm{~mm}$; field of view, $280 \times 280 \mathrm{~mm}$; and scan time, 39 seconds and three-dimensional-TE, $4 \mathrm{~ms}$; TR, 8 ms; FA, 75; field of view, $350 \times 350 \times 280$; matrix, $208 \times$ $208 \times 122$; and scan time, 8 minutes 6 seconds. Under the NL condition, the talocrural joint was positioned at a $90^{\circ}$ angle to the long axis of the tibia. Under the FW condition, participants were positioned in a two-legged stance and instructed to stand with pressure equally distributed on the heel and anterior plantar sole. Under both NL and FW conditions, the foot was oriented vertically to the scanner bed, with the participants' feet at the same width apart as that in a relaxed standing position [2]. A careful examination was conducted to ensure that there would be no differences in foot positions between the conditions.

\section{Image Analysis}

For image analysis, we used commercially available software (ITK SNAP, www.itksnap.org) [21]. The hindfoot alignment view (HAV), navicular bone height $(\mathrm{NH})$, talus bone height (TH), medial navicular bone position (MNP), and medial talus bone position (MTP) were measured. The methods for obtaining HAV, NH, TH, MNP, and MTP measurements have previously been described in detail $[2,22-$ 24].

To measure the HAV, we used coronal images of the foot, in which the most central image through the distal tibial shaft was shown. The image was identified as the one in which the tibial shaft diameter was maximal, with a sharply defined tibial cortex [2,22]. First, we drew two lines which connects two pairs of points on the cortex of the distal tibia. We set the lower line above the distal tibial spread adjacent to the ankle joint and the upper line approximately $2.0 \mathrm{~cm}$ above the lower line. We drew the distal tibial shaft axis which connects the midpoints of two lines $<$ Figure $1>$ (a). Then, we scrolled through the coronal images to find the lowest point of the calcaneus $<$ Figure $1>$ (b). Finally, we measured the distance from the tibial axis to the lowest point of the calcaneus as the apparent moment arm < Figure $1>(\mathbf{c})$. Positive values signified hindfoot valgus, and negative values signified hindfoot varus.

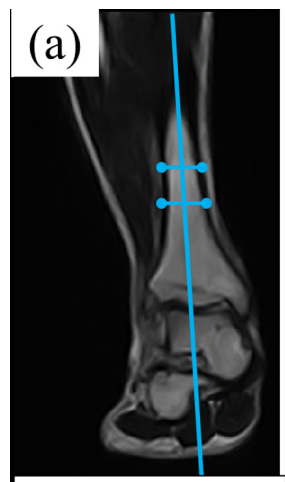

The tibial mid-diaphyseal line
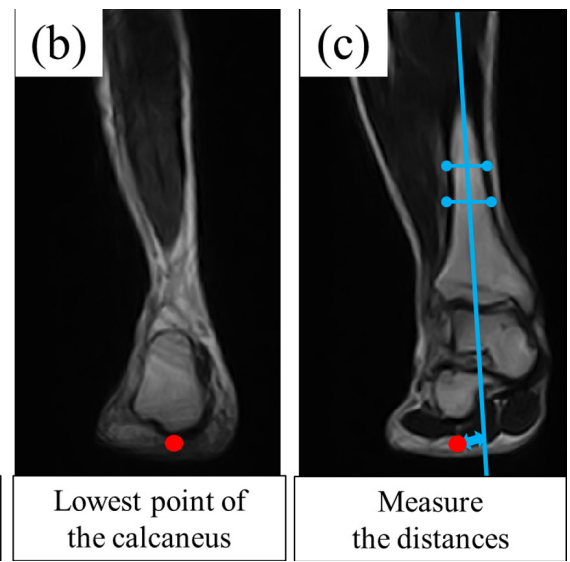

Figure 1. Measuring the hindfoot alignment view (HAV): (a) We drew two lines which connects two pairs of points on the cortex of the distal tibia. The lower line is set above the distal tibial spread adjacent to the ankle joint and the upper line approximately $2.0 \mathrm{~cm}$ above the lower line. Then, we drew the tibial mid-diaphyseal line which connects the midpoint of two lines. (b) We marked the lowest point of the calcaneus bone; and (c) we measured the HAV as the distance between the tibial mid-diaphyseal line to the lowest point of the calcaneus bone.

We analyzed NH, TH, MNP, and MTP values with reference to the methods of a previous study [2,24]. For measurement of $\mathrm{NH}(\mathrm{TH})$, coronal images of the foot were used. We chose the coronal image in which the lowest point of the navicular (talus) bone was visualized in order to determine the measuring point. Based on these images, the $\mathrm{NH}(\mathrm{TH})$ was measured as the distance from the floor to the lowest point of the navicular (talus) bone $<$ Figure $2>$.

For measurement of MNP (MTP), axial images of the foot were used. First, we chose the axial image in which the head of the fifth metatarsal bone was visualized, as well as the axial image in which the most caudal point of the calcaneus bone 


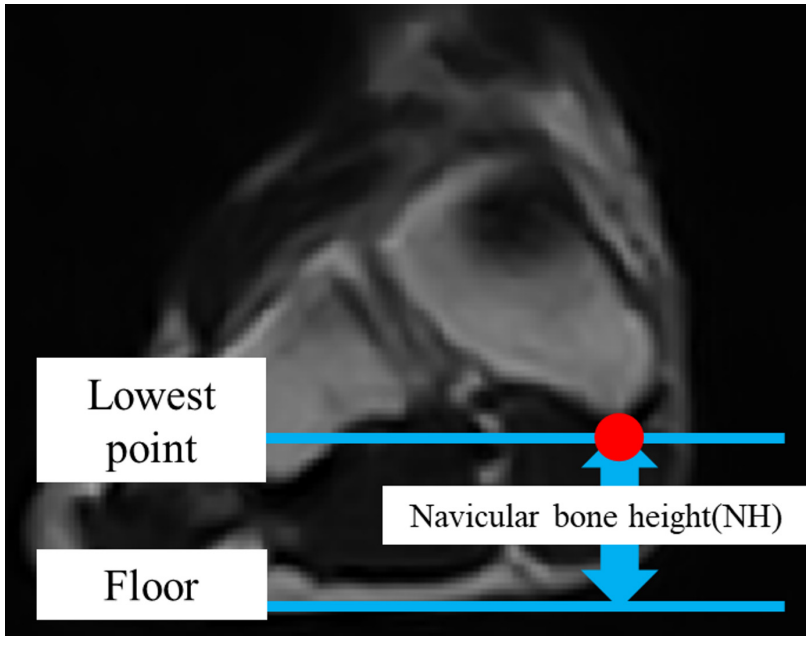

Figure 2. Measuring the navicular bone height (NH). We measured the $\mathrm{NH}$ as the distance from the floor to the lowest point of the navicular bone.

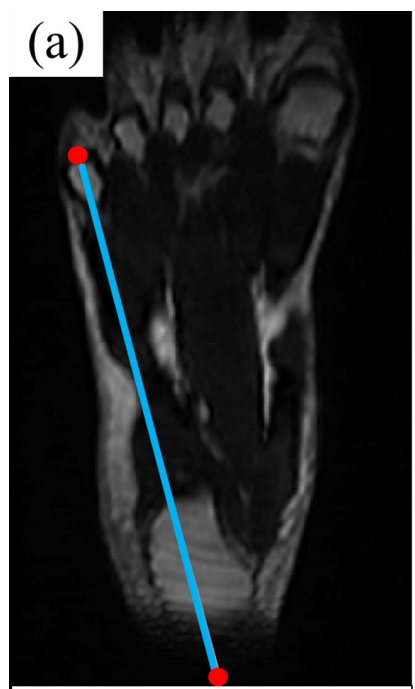

Determine the baseline

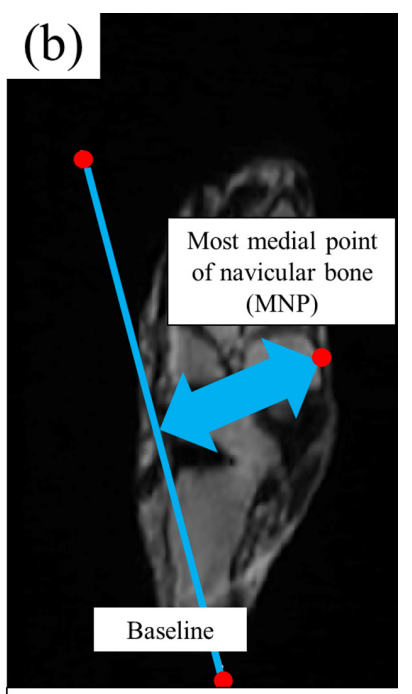

Medial navicular bone position(MNP)
Figure 3. Measuring the medial navicular bone position (MNP): (a) First, we determined the baseline for measurements of MNP, which connects the most caudal point of the calcaneus bone and the head of the fifth metatarsal bone, and (b) we measured the MNP as the distance between the baseline and the most medial point of the navicular bone.

was visualized. Based on these two images, the baseline for measurement of MNP (MTP) was determined $<$ Figure $3>$ (a). Finally, the MNP (MTP) was measured as the distance from the baseline to the most medial point of the navicular (talus) bone $<$ Figure $3>(\mathbf{b})$.

\section{Calculation of Navicular and Talus Bone Position Changes}

We calculated changes in the HAV, NH, TH, MNP, and
MTP ( $\triangle \mathrm{HAV}, \Delta \mathrm{NH}, \Delta \mathrm{TH}, \Delta \mathrm{MNP}$, and $\Delta \mathrm{MTP})$ between the $\mathrm{NL}$ and FW conditions (FW-NL).

The total weight-bearing response of the navicular and talus bones can be represented by both the distance and the direction. In this study, distance was represented using the total positional changes of the navicular and talus bones ( $\triangle \mathrm{TPCN}, \triangle \mathrm{TPCT}$ ), which is a combination of the vertical and medial displacements of these bones. $\triangle \mathrm{TPCN}$ and $\triangle \mathrm{TPCT}$ were calculated from vertical and medial displacements using the Pythagorean theorem, as follows: [2]

$$
\begin{aligned}
& \triangle \mathrm{TPCN}=\sqrt{\left(\Delta N H^{2}+\Delta M N P^{2}\right)} \\
& \triangle \mathrm{TPCT}=\sqrt{\left(\Delta T H^{2}+\Delta M T P^{2}\right)}
\end{aligned}
$$

Direction was represented using the total positional angle changes of the navicular and talus bones (TPACN, TPACT), which is the ratio of the vertical-to-medial displacements of these bones. TPACN and TPACT were estimated using these equations: [2]

$$
\begin{aligned}
& \text { TPACN }=\tan ^{-1} \frac{\Delta N H}{\Delta M N P} \\
& \text { TPACT }=\tan ^{-1} \frac{\Delta T H}{\Delta M T P}
\end{aligned}
$$

\section{Statistical Analyses}

Measurements for HAV, NH, TH, MNP, MTP, $\triangle \mathrm{HAV}$, $\triangle \mathrm{NH}, \triangle \mathrm{TH}, \triangle \mathrm{MNP}, \triangle \mathrm{MTP}, \triangle \mathrm{TPCN}$, and $\triangle \mathrm{TPCT}$ are presented in millimeters. TPACT and TPACN are presented in degrees $\left({ }^{\circ}\right)$. We used the Shapiro-Wilk test to confirm the normality. Normality was not observed in the HAV alone on NL condition when a medial heel wedge was used. However, analysis of variance (ANOVA) is a robust statistic [25,26]; thus, we performed a two-way (two loading conditions [NL and FW] $\times$ two insole types [flat insole and medial heel wedge]) repeated-measures ANOVA for the HAV, NH, TH, MNP, and MTP. If significant interactions were found, the Bonferroni test was performed as a post hoc test for the NH, TH, MNP, and MTP and non-parametric Wilcoxon signed-rank tests was performed as a post hoc test for HAV. All statistical analyses were conducted using SPSS software, version 26.0, 
for Windows (IBM SPSS Japan Inc., Japan).

\section{Results}

$<$ Table $1>$ shows the HAV, NH, TH, MNP, and MTP values under NL and FW conditions. HAV showed a significant loading conditions $\times$ insole interaction $\left(F=7.51 ; P=0.01 ; \eta^{2}\right.$ $=0.24)$. Under both NL and FW conditions, the HAV was significantly smaller on the medial heel wedge than on the flat insole (NL, $P<0.01$; FW, $P<0.01$ ). Additionally, NH showed a significant loading conditions $\times$ insole interaction $(F=12.61$; $\left.P<0.01 ; \eta^{2}=0.35\right)$. Under the NL condition, no significant differences were observed between any insole types $(P=0.72)$. Under the FW condition, the $\mathrm{NH}$ was larger on the medial heel wedge than on the flat insole $(P<0.01)$. No significant interactions were observed for TH, MNP, or MTP (TH, $F=$ 2.80, $P=0.11, \eta^{2}=0.11 ; \mathrm{MNP}, F=3.56, P=0.07, \eta^{2}=0.13$; and MTP, $F=2.23, P=0.15, \eta^{2}=0.09$ ).

Table 1. The position of foot arch bones in non-loading (NL) and full weight-bearing (FW) conditions. $(n=25)$

\begin{tabular}{|c|c|c|c|c|c|}
\hline & & \multicolumn{2}{|c|}{ Flat insole } & \multicolumn{2}{|c|}{ Medial heel wedge } \\
\hline & & Average & SD & Average & SD \\
\hline \multirow{2}{*}{$\begin{array}{l}\text { Hindfoot alignment } \\
\text { view } \\
\text { (HAV) }(\mathrm{mm})\end{array}$} & Non-loading (NL) & $\begin{array}{l}-2.58^{*} \\
M:-2.47\end{array}$ & $\begin{array}{l}4.77 \\
R:(-13.04,6.31)\end{array}$ & $\begin{array}{l}-5.69 \\
M:-4.13\end{array}$ & $\begin{array}{l}4.94 \\
R:(-20.55,1.65)\end{array}$ \\
\hline & Full weight-bearing (FW) & $\begin{array}{l}5.35^{*} \\
M: 5.51\end{array}$ & $\begin{array}{l}5.83 \\
R:(-4.21,20.00)\end{array}$ & $\begin{array}{l}1.84 \\
M: 1.88\end{array}$ & $\begin{array}{l}5.53 \\
R:(-12.04,12.67)\end{array}$ \\
\hline \multirow{3}{*}{$\begin{array}{l}\text { Navicular bone height } \\
(\mathrm{NH})(\mathrm{mm})\end{array}$} & Non-loading (NL) & 37.56 & 6.30 & 37.70 & 6.74 \\
\hline & Full weight-bearing (FW) & $26.15^{*}$ & 5.14 & 28.01 & 5.08 \\
\hline & $\Delta \mathrm{NH}=\mathrm{FW}-\mathrm{NL}$ & -11.41 & 0.64 & -9.69 & 0.57 \\
\hline \multirow[b]{2}{*}{$\begin{array}{l}\text { Talus bone height } \\
\text { (TH) }(\mathrm{mm})\end{array}$} & Non-loading (NL) & 47.91 & 5.11 & 48.74 & 5.59 \\
\hline & Full weight-bearing (FW) & 39.39 & 5.11 & 41.06 & 4.82 \\
\hline \multirow{3}{*}{$\begin{array}{l}\text { Medial navicular bone } \\
\text { position } \\
(\mathrm{MNP})(\mathrm{mm})\end{array}$} & Non-loading (NL) & 52.60 & 5.61 & 53.15 & 5.49 \\
\hline & Full weight-bearing (FW) & 56.90 & 5.15 & 56.47 & 5.46 \\
\hline & $\Delta \mathrm{MNP}=\mathrm{FW}-\mathrm{NL}$ & 4.31 & 0.54 & 3.32 & 0.47 \\
\hline \multirow{3}{*}{$\begin{array}{l}\text { Medial talus bone } \\
\text { position } \\
\text { (MTP) }(\mathrm{mm})\end{array}$} & Non-loading (NL) & 43.48 & 6.01 & 43.91 & 5.87 \\
\hline & Full weight-bearing (FW) & 49.90 & 5.46 & 49.44 & 5.75 \\
\hline & $\Delta \mathrm{MTP}=\mathrm{FW}-\mathrm{NL}$ & 6.42 & 0.64 & 5.53 & 0.60 \\
\hline
\end{tabular}

*: Flat insole vs Medial heel wedge, $\mathrm{P}<0.05 ; \mathrm{M}=$ median; $\mathrm{R}=$ range

Table 2. The $\triangle T P C N, \triangle T P C T$, TPACN, and TPACT are shown $(n=25)$.

\begin{tabular}{|c|c|c|c|c|c|c|}
\hline & \multicolumn{2}{|c|}{ Flat insole } & \multicolumn{2}{|c|}{ Medial heel wedge } & \multicolumn{2}{|c|}{ Change (Medial heel wedge - flat insole) } \\
\hline & Average & SD & Average & SD & Average & $95 \% \mathrm{Cl}$ \\
\hline$\triangle T P C N(\mathrm{~mm})$ & $12.41 *$ & 3.48 & 10.43 & 3.13 & -1.98 & $(-2.96,-1.01)$ \\
\hline$\triangle \mathrm{TPCT}(\mathrm{mm})$ & 10.90 & 3.62 & 9.74 & 2.90 & -1.17 & $(-2.45,0.11)$ \\
\hline $\operatorname{TPACN}\left({ }^{\circ}\right)$ & 20.14 & 10.83 & 18.05 & 11.63 & -2.09 & $(-7.85,3.67)$ \\
\hline TPACT $\left({ }^{\circ}\right)$ & 36.02 & 13.09 & 34.90 & 14.04 & -1.12 & $(-7.05,4.82)$ \\
\hline
\end{tabular}

*: Flat insole vs Medial heel wedge, $\mathrm{P}<0.05$

$\triangle \mathrm{TPCN}=$ total positional change of the navicular bone

$\triangle T P C T=$ total positional change of the talus bone

TPACN = total positional angle change of the navicular bone

TPACT $=$ total positional angle change of the talus bone 
$<$ Table $2>$ shows the $\triangle \mathrm{TPCN}, \triangle \mathrm{TPCT}$, TPACN, and TPACT. The $\triangle \mathrm{TPCN}$ was significantly smaller on the insole with the medial heel wedge than on the flat insole $(P<0.01)$. No significant differences were observed for the $\triangle \mathrm{TPCT}$, TPACN, or TPACT $(\triangle T P C T: P=0.07$, TPACN: $P=0.46$, and TPACT: $P=0.70)$.

\section{Discussion}

In our study, we evaluated the effects of a medial heel wedge on the weight-bearing response of hindfoot valgus and the total weight-bearing responses of the navicular and talus bones using pMRI. The present study contains salient findings that require discussion. First, under both NL and FW conditions, the HAV was smaller on the medial heel wedge than on the flat insole. Second, under the FW condition, the $\mathrm{NH}$ was larger on the medial heel wedge than on the flat insole. Finally, the $\triangle \mathrm{TPCN}$ was significantly smaller on the medial heel wedge than on the flat insole.

Our results showed that a medial heel wedge reduced HAV values under both NL and FW conditions, indicating that the use of a medial heel wedge redirects the reaction forces acting on the plantar foot from a more lateral to a more medial location. Kirby et al. theoretically reported that redirecting the reaction forces acting on the plantar foot from a more lateral to a more medial location would decrease the hindfoot valgus moment [27]. Therefore, the use of a medial heel wedge may reduce the hindfoot valgus moment [28,29]. Excessive hindfoot valgus and hindfoot valgus moments have been linked to a risk of lower-limb overuse injuries [30,31]. Our findings indicate why the prescription of a heel wedge may be effective for the management of musculoskeletal disorders of the lower limb.

Furthermore, our results showed that when the heel wedge is inserted, $\mathrm{NH}$ increased under the FW condition and $\triangle$ TPCN decreased. It means that the use of a medial heel wedge plays a role in limiting the $\triangle \mathrm{TPCN}$ by increasing the NH under the FW condition. Previous studies have reported that the movement of a hindfoot valgus correlates with the movements of the navicular and talus bones [2]. Therefore, a change in $\triangle \mathrm{TPCN}$ (i. e., $\triangle \mathrm{TPCN}$ on medial heel wedge condition - $\triangle \mathrm{TPCN}$ on flat insole condition) may be caused by a change in $\triangle \mathrm{HAV}$ (i.e., $\triangle \mathrm{HAV}$ on medial heel wedge condition - $\triangle \mathrm{HAV}$ on flat insole condition). We examined the relationship between the amount of change in $\triangle \mathrm{HAV}$ (the average \pm SD values was $-0.40 \pm 0.72$ ) and the amount of change in $\triangle \mathrm{TPCN}$ (the average \pm SD values are in Table 2) using a Pearson's correlation coefficient across all subjects, without finding a significant correlation $(r=0.04, P$-value $=$ $0.84)$. This result suggests that other factors, such as changes in plantar foot pressure due to the medial heel wedge, may limit the $\triangle \mathrm{TPCN}$ [9]. Some previous studies have reported that increased mobility of the navicular bone is related to a risk of lower limb injuries [8,32]. Therefore, stabilization of the total weight-bearing response of the navicular bone by the prescription of a medial heel wedge may be effective for management of musculoskeletal disorders of the lower limb.

Our results also showed that the use of a medial heel wedge did not affect the TPACN nor the TPACT. When an axial load is applied, the rotation of the talonavicular joint around the subtalar joint axis has been reported to occur simultaneously with the weight-bearing response of the hindfoot valgus [33]. In addition, the navicular and talus bones slide down the subtalar articular surfaces as a rigid unit [33]. Then, the talus bone pushes out the navicular bone vertically and medially [33]. Based on this information, it is possible that the TPACN and TPACT could be affected by the subtalar joint axis location $[2,27,33,34]$ and the talonavicular joint alignment [33]. Our results indicate that the use of a heel wedge may not change these structures, which may explain why the use of a medial heel wedge did not affect the TPACN nor the TPACT in our study.

This study had a few limitations. The present study was conducted only in healthy adult males, though age, gender, and foot function may also affect the weight-bearing response of foot-arch bones [8,32,35-37]. Further, we measured only the right foot of participants. The characteristics of the foot, such as the dominant leg, may affect our result [38]. Therefore, we should examine the effects of these factors in future studies.

We applied only one heel wedge and flat insole to clarify the effects of a medial heel wedge on the weight-bearing response of hindfoot valgus and the total weight-bearing 
responses of the navicular and talus bones. The participants' foot morphologies may vary, and the pattern of combination of insole types and foot morphology may affect our results [3941]. The relationship between the foot characteristics and the insole type should be examined in future studies. In contrast, several previous studies reported the effectiveness of a medial heel wedge similar in shape to the one we used [10,42-45]. We believe that these findings support our study.

We performed an MRI immediately after the participants wore the insole; hence, our results could have indicated the temporary effect of a medial heel wedge. Conversely, we could not consider the effects of acclimatization of the insole insertion and alignment over time. The effects of acclimatization of the insole insertion and alignment over time need to be discussed in future studies [46].

Moreover, we could not study the effects of medial heel wedge on the navicular and talus bone positions during exercise because measuring the weight-bearing responses of foot-arch bones during exercise has been challenging. To study the effects of medial heel wedge on the navicular and talus bone positions during exercise in future studies would be more realistic knowledge for clinical management [23]. However, we believe that our results are significant for the management of musculoskeletal injuries.

\section{Conclusions}

In conclusion, the present study suggested that a medial heel wedge decreases HAV values under both NL and FW conditions and stabilizes the total weight-bearing response of the navicular bone.

\section{Acknowledgments}

This work was partially supported by JSPS KAKENHI [Grant Number 19H05730].

\section{Conflicts of Interest}

The authors declare no conflict of interest.

\section{References}

1. McKenzie J. The foot as a half-dome. Br Med J. 1955; 1(4921):1068-1069.

2. Maruyama M, Yozu A, Okamoto Y, Shiraki H. Relationship between total weight-bearing response of the navicular and talus bones and weight-bearing response of hindfoot valgus in normal foot arch. J Phys Fitness Sports Med. 2021; 10(2):75-84.

3. Lundberg A, Svensson OK, Bylund C, Goldie I, Selvik G. Kinematics of the Ankle/Foot Complex-Part 2: Pronation and Supination. Foot Ankle. 1989; 9(5):248253.

4. Maharaj JN, Cresswell AG, Lichtwark GA. Subtalar Joint Pronation and Energy Absorption Requirements During Walking are Related to Tibialis Posterior Tendinous Tissue Strain. Sci Rep. 2017; 7(1):17958.

5. Ker RF, Bennett MB, Bibby SR, Kester RC, Alexander RM. The spring in the arch of the human foot. Nature. 1987; 325(7000):147-149.

6. Ogon M, Aleksiev AR, Pope MH, Wimmer C, Saltzman CL. Does Arch Height Affect Impact Loading at the Lower Back Level in Running? Foot Ankle Int. 1999; 20(4):263-266.

7. Maharaj JN, Cresswell AG, Lichtwark GA. Foot structure is significantly associated to subtalar joint kinetics and mechanical energetics. Gait Posture. 2017; 58:159-165.

8. Zhang Y-J, Du J-Y, Chen B, Jin R-L, Hu J-G, Lin X-J. Correlation between three-dimensional medial longitudinal arch joint complex mobility and medial arch angle in stage II posterior tibial tendon dysfunction. J Foot Ankle Surg. 2019; 25(6):721-726.

9. Gheluwe B, Dananberg H. Changes in Plantar Foot Pressure with In-Shoe Varus or Valgus Wedging. J Am Podiatr Med Assoc. 2004; 94:1-11.

10. Shih Y-F, Wen Y-K, Chen W. Application of wedged foot orthosis effectively reduces pain in runners with pronated foot: A randomized clinical study. Clin Rehabil. 2011; 25:913-923.

11. Pascual Huerta J, Ropa Moreno JM, Kirby KA, García Carmona FJ, Orejana García AM. Effect of 7-degree 
rearfoot varus and valgus wedging on rearfoot kinematics and kinetics during the stance phase of walking. J Am Podiatr Med Assoc. 2009; 99(5):415-421.

12. Power V, Clifford AM. The Effects of Rearfoot Position on Lower Limb Kinematics during Bilateral Squatting in Asymptomatic Individuals with a Pronated Foot Type. J Hum Kinet. 2012; 31:5-15.

13. Hajizadeh M, Desmyttere G, Carmona J-P, Bleau J, Begon M. Can foot orthoses impose different gait features based on geometrical design in healthy subjects? A systematic review and meta-analysis. Foot (Edinb). 2020; 42:101646.

14. Mündermann A, Nigg BM, Humble RN, Stefanyshyn DJ. Foot orthotics affect lower extremity kinematics and kinetics during running. Clin Biomech. 2003; 18(3):254-262.

15. Menz HB. Foot orthoses: how much customisation is necessary? J Foot Ankle Res. 2009; 2:23.

16. McPoil TG, Vicenzino B, Cornwall MW, Collins N, Warren M. Reliability and normative values for the foot mobility magnitude: a composite measure of vertical and mediallateral mobility of the midfoot. J Foot Ankle Res. 2009; $2: 6$.

17. Khamis S, Yizhar Z. Effect of feet hyperpronation on pelvic alignment in a standing position. Gait Posture. 2007; 25(1):127-134.

18. Ghasemi MS, Koohpayehzadeh J, Kadkhodaei H, Ehsani AA. The effect of foot hyperpronation on spine alignment in standing position. Med J Islam Repub Iran. 2016; 30:466.

19. Mattingly B, Talwalkar V, Tylkowski C, Stevens DB, Hardy PA, Pienkowski D. Three-dimensional in vivo motion of adult hind foot bones. J Biomech. 2006; 39(4):726-733.

20. Lamm BM, Stasko PA, Gesheff MG, Bhave A. Normal Foot and Ankle Radiographic Angles, Measurements, and Reference Points. J Foot Ankle Surg. 2016; 55(5):991998.

21. Yushkevich PA, Piven J, Hazlett HC, et al. User-guided 3D active contour segmentation of anatomical structures: Significantly improved efficiency and reliability. NeuroImage. 2006; 31(3):1116-1128.
22. Buber N, Zanetti M, Frigg A, Saupe N. Assessment of hindfoot alignment using MRI and standing hindfoot alignment radiographs (Saltzman view). Skeletal Radiol. 2018; 47(1):19-24.

23. Johannsen F, Hansen P, Stallknecht S, et al. Can positional MRI predict dynamic changes in the medial plantar arch? An exploratory pilot study. J Foot Ankle Res. 2016; 9:35.

24. Hansen P, Johannsen FE, Hangaard S, et al. Navicular bone position determined by positional MRI: a reproducibility study. Skeletal Radiol. 2016; 45(2):205211.8

25. Glass GV, Peckham PD, Sanders JR. Consequences of Failure to Meet Assumptions Underlying the Fixed Effects Analyses of Variance and Covariance. Review of Educational Research. 1972; 42(3):237-288.

26. Blanca MJ, Alarcón R, Arnau J, Bono R, Bendayan R. Nonnormal data: Is ANOVA still a valid option? Psicothema. 2017; 29(4):552-557.

27. Kirby KA. Subtalar joint axis location and rotational equilibrium theory of foot function. J Am Podiatr Med Assoc. 2001; 91(9):465-487.

28. Jafarnezhadgero A, Alavi-Mehr SM, Granacher U. Effects of anti-pronation shoes on lower limb kinematics and kinetics in female runners with pronated feet: The role of physical fatigue. PLoS One. 2019; 14(5):e0216818.

29. Maharaj JN, Cresswell AG, Lichtwark GA. The Immediate Effect of Foot Orthoses on Subtalar Joint Mechanics and Energetics. Med Sci Sports Exerc. 2018; 50(7):14491456.

30. Tiberio D. The effect of excessive subtalar joint pronation on patellofemoral mechanics: a theoretical model. J Orthop Sports Phys Ther. 1987; 9(4):160-165.

31. Niki H, Ching RP, Kiser P, Sangeorzan BJ. The effect of posterior tibial tendon dysfunction on hindfoot kinematics. Foot Ankle Int. 2001; 22(4):292-300.

32. Bandholm T, Boysen L, Haugaard S, Zebis MK, Bencke J. Foot Medial Longitudinal-Arch Deformation During Quiet Standing and Gait in Subjects with Medial Tibial Stress Syndrome. J Foot Ankle Surg. 2008; 47(2):89-95. 33. Ito K, Hosoda K, Shimizu M, et al. Three-dimensional 
innate mobility of the human foot bones under axial loading using biplane X-ray fluoroscopy. R Soc Open Sci. 2017; 4(10):171086.

34. Bruckner J. Variations in the Human Subtalar Joint. J Orthop Sports Phys Ther. 1987; 8(10):489-494.

35. Faria A, Gabriel R, Abrantes J, Brás R, Moreira H. The relationship of body mass index, age and triceps-surae musculotendinous stiffness with the foot arch structure of postmenopausal women. Clin Biomech. 2010; 25(6):588-593.

36. Fukano M, Fukubayashi T. Gender-based differences in the functional deformation of the foot longitudinal arch. Foot (Edinb). 2012; 22(1):6-9.

37. Fukano M, Fukubayashi T, Banks SA. Sex differences in three-dimensional talocrural and subtalar joint kinematics during stance phase in healthy young adults. Hum Mov Sci. 2018; 61:117-125.

38. Van Melick N, Meddeler BM, Hoogeboom TJ, Nijhuis-van der Sanden MWG, van Cingel REH. How to determine leg dominance: The agreement between self-reported and observed performance in healthy adults. PLoS One. 2017; 12(12):e0189876-e0189876.

39. Van Gheluwe B, Dananberg HJ. Changes in plantar foot pressure with in-shoe varus or valgus wedging. J Am Podiatr Med Assoc. 2004; 94(1):1-11.

40. Goske S, Erdemir A, Petre M, Budhabhatti S, Cavanagh PR. Reduction of plantar heel pressures: Insole design using finite element analysis. J Biomech. 2006; 39(13):23632370 .
41. Bonanno DR, Zhang CY, Farrugia RC, et al. The effect of different depths of medial heel skive on plantar pressures. J Foot Ankle Res. 2012; 5:20.

42. Joseph M, Tiberio D, Baird JL, et al. Knee valgus during drop jumps in National Collegiate Athletic Association Division I female athletes: the effect of a medial post. Am J Sports Med. 2008; 36(2):285-289.

43. Anand UA, Santiago B, Beuke P, et al. A Study to Analyse the Effectiveness of Physical Therapy and Wedged Foot Orthotic Devices on Pain in Runners with Pronated Foot. Acta Sci Orthop. 2019; 2:40-43.

44. Chang HY, Chang YC, Cheng SC, Wang CH. The effectiveness of rearfoot medial wedge intervention on balance for athletes with chronic ankle instability. Medicine (Baltimore). 2019; 98(26):e16217.

45. Bakhtiari F, Bahramizadeh M, Safaeepour Z, Vahedi M. Effects of Custom-mold Insole by Medial Heel Skive Technique on Physical Function in Flexible Flat Foot. Iran Rehabil J. 2021; 19(2):181-188.

46. Jafarnezhadgero A, Madadi-Shad M, Alavi-Mehr SM, Granacher U. The long-term use of foot orthoses affects walking kinematics and kinetics of children with flexible flat feet: A randomized controlled trial. PLoS One. 2018; 13(10):e0205187.1.

47. McKenzie J. The foot as a half-dome. Br Med J. 1955; 1(4921):1068-1069. 